\title{
Use of Laboratory Decision System as a Test Utilization Management Tool in Clinical Settings, Current and Future Perspectives
}

\author{
Lucas Leblow ${ }^{1}$, Timothy Hamill², and Safedin H Beqaj,* \\ ${ }^{1}$ Laboratory Decision System, Medical Database, Inc, California, USA \\ ${ }^{2}$ UCSF Department of Laboratory Medicine, San Francisco, California, USA \\ ${ }^{3}$ Universal Diagnostic Laboratories, Van Nuys, California, USA
}

*Corresponding author: Safedin H Beqaj, Universal Diagnostic Laboratories, Van Nuys, California, USA, E-mail: sajo@udlab.com

Received: 14 Jul, 2019 | Accepted: 30 Aug, 2019 | Published: 05 Sep, 2019

Citation: Leblow L, Hamill T, Beqaj SH (2019) Use of Laboratory Decision System as a Test Utilization Management Tool in Clinical Settings, Current and Future Perspectives. J Clin Lab Med 4(1): dx.doi.org/10.16966/2572-9578.128

Copyright: ( 2019 Leblow L, et al. This is an open-access article distributed under the terms of the Creative Commons Attribution License, which permits unrestricted use, distribution, and reproduction in any medium, provided the original author and source are credited.

\begin{abstract}
Managing laboratory test utilization has been a growing problem for the healthcare industry for a long time. With an ever-increasing number of tests, especially in the area of molecular genetics where per test costs are very high, inappropriate utilization is creating a financial burden on healthcare overall. Several large healthcare institutions have made efforts to solve this problem and have developed their own test utilization management approaches. These include, physician education, providing test pricing information, utilizing reminders in Computerized Provider Order Entry (CPOE) systems and/or setting up committees to authorize the use of expensive or complex tests. While these approaches have achieved some minor success in curbing test overutilization and generating cost savings, a robust automated Clinical Laboratory Decision Support System has still been sorely lacking. We present, here, a newly developed Laboratory Decision System, LDS ${ }^{\mathrm{TM}}$ as a potential method to address test utilization management in clinical settings. A study of provider and payer use of LDS ${ }^{\mathrm{TM}}$ revealed significant improvements in test ordering and management on both sides: a significant reduction in unnecessary tests from the provider's side and measurable improvement in medical necessity checks from the payer's side.
\end{abstract}

\section{Introduction}

Currently, physicians are challenged by a lack of access to centralized information regarding thousands of available clinical laboratory tests [1]. A study conducted by the Common wealth Fund Survey of Public Views of the U.S. Health Care System showed that over $23 \%$ of tests ordered by physicians had previously been performed. Such duplication increases the cost of care while further delaying or confusing the patient's diagnosis and care [1,2]. Physician uncertainty related to appropriate laboratory utilization was studied by the Center for Disease Control (CDC) in 2011. The study surveyed 1,768 primary care physicians in the U.S and demonstrated that $14.7 \%$ had uncertainty in selecting and ordering the correct test and $8.3 \%$ had difficulty interpreting tests [3]. When these statistics are applied to over 300 million patient laboratory visits a year in the U.S, inappropriate test ordering and interpretation potentially impacts 23 million patients annually [3]. Such inappropriate test utilization has further downstream clinical and cost impact on our overall healthcare system. The survey also indicated that more than three-quarters of physician respondents indicated that consulting an expert, checking e-references or specialist referrals was helpful in reducing uncertainty in ordering and interpreting lab tests [3].

For these and other compelling reasons, payers, particularly Medicare, are beginning to require standardization of practices and methods for reducing wasteful testing. A study published by the American College of Physicians (ACP), in 2012, stemming the Tide of over treatment in U.S. Healthcare, explains "the impact of unnecessary tests on healthcare cost totals up to $\$ 250$ billion a year" [4]. Timely, relevant and actionable data is needed for doctors to accurately order tests, and for labs and other rendering providers to deliver services efficiently and get paid in a timely manner. Further, the rapid growth in molecular and genetic testing (welcomed new tools for diagnosis and disease management) poses a challenge for both healthcare providers and for commercial payers regarding proper utilization of these specialized tests. Given their relatively high cost, inappropriate use of these tests represents an additional financial burden on an already over-taxed healthcare system $[2,4,5]$.

Since laboratory testing provides $70-85 \%$ of the objective data upon which physicians base their diagnoses and treatments, laboratory diagnostics has become the single highest-volume medical activity in the U.S., with an estimated 4-5 billion tests performed annually [5]. Inappropriate testing consists of both over- and underutilization, which together can dramatically increase healthcare costs. Overutilization refers to tests that are ordered when not clinically indicated, while underutilization refers to tests that are clinically indicated but not ordered. A Harvard Medical School 15- 
year meta-analysis indicated that overutilization and underutilization of laboratory tests occurs $20.6 \%$ and $44.8 \%$ of the time respectively [6]. Inappropriate testing may lead to incorrect or delayed diagnoses and treatments, which negatively impact patient recovery time and associated costs. Test overutilization often increases the likelihood of false-positive results which can result in incorrect diagnoses, additional inappropriate testing, increased costs, and adverse outcomes due to unwarranted intervention [6,7]. Additionally, a consequence of ordering tests which are not indicated often can be the failure to order tests which are clinically 'indicated', further compounding the problem. Test underutilization can contribute to an increase in morbidity due to delayed or missed diagnoses and, paradoxically, can lead to downstream overutilization, again resulting in higher costs and poorer patient care [2,7].

With industry and governmental shifts from traditional fee-forservice to value-based care, over/underutilization is a critical issue that affects the bottom line with respect to increased costs and poor clinical outcomes. In order to meet the goals of modern healthcare, medical providers and insurance payers are searching for solutions that will help in making optimal decisions in the selection of diagnostic tests and ensuring the appropriate utilization of laboratory resources.

\section{Current Approach to Test Utilization Problem}

There have been several efforts by large healthcare institutions to counter test utilization problems. However, it is worth listing the main factors influencing ordering behavior: ease of ordering in CPOE, ignorance of test characteristics, peer or supervisor pressure to be extremely thorough, fear of litigation, impatience, desire for certainty of diagnosis, financial incentives, and patient demand [2,5,8,9]. In general, the overall management of these factors requires new tools, education, changes in habits and reward feedback loops [8,10-12]. Two important studies that have attempted to analyze and solve the problem of test utilization are the Veterans Affairs Hospital study [13] and the Massachusetts General Hospital (MGH) study $[8,14]$. The Veterans Affairs Hospital study managed test utilization by implementing a modified and improved laboratory information system that allowed them to setup special rules, restrictions, reminders and notifications, which lead to reduction in unnecessary tests and decreased cost [13]. The MGH study developed a laboratory utilization management program that required committee review and approval for test orders and acted as a 'governor' against practitioners ordering 'inappropriate' tests. This method is predictably costly as it necessitates the 'handson' participation of expensive experts including pathologists and genetic counselors in decision making and does not result in timely responses $[2,5,8,14]$. Over burdened clinicians cannot afford to spend large amounts of time tracking down a pathologist for an answer that they often require immediately. Several other studies have used similar strategies to reduce unnecessary test ordering and cost reduction, especially with respect to sending out tests which are often poorly reimbursed, and, therefore, create financial burdens for many small and large hospitals $[8,15,16]$.

\section{Future Approach to Test Utilization Problem}

\section{Clinical decision support system}

Selecting appropriate medical tests is an ongoing and growing problem in many specialties including radiology, cardiology, Pulmonology and pharmacology. Since radiology diagnostic imaging is more costly than laboratory testing, the U.S. government has approved a reimbursement reward system for insurance providers that utilize a Clinical Decision Support System (CDSS) to improve imaging utilization and, thereby, decrease unnecessary orders and cost [15]. For example, there is a 2015 "Advanced Imaging Bill" which mandates that government-approved imaging services will only be reimbursed if the insurance claim confirms that appropriate-use criteria was consulted or a CDSS was used $[17,18]$. The Bill also recommends use of CDSS for other diagnostic test ordering, if available. Accordingly, CDSS's are currently available for cardiology, medication management, oncology and urology. These developments strongly indicate that there also is a substantial need for a laboratory CDSS to aid healthcare providers in selecting and ordering laboratory tests and reducing inappropriate testing [11,12]. Currently, there are some partially developed and semi-manual lab CDSS's that help physicians order laboratory tests; however, these modules are provider-driven and require inconvenient interactive user questions to access the information needed [9$11,19,20]$. In addition, these systems do not provide any scoring system for tests based on medical evidence, clinical relevancy and medical necessity as used by radiology CDSS's.

\section{Laboratory decision support system}

To address these challenges, Medical Database, Inc (MDB) has developed a laboratory decision support platform that includes an easy-to-use test ordering and utilization management application, also known as the Laboratory Decision System or LDS ${ }^{\mathrm{TM}}$. This platform gives healthcare providers access to a systematic and in-depth resource to assist in selecting and ordering the most appropriate laboratory tests using evidence-based guidelines and industry best practices. This data base is designed to aid physicians in better understanding, selecting, ordering, and interpreting the most relevant lab tests for their patient's condition. LDS $^{\mathrm{TM}}$ also incorporates a proprietary "MDB Ranking System" which supports healthcare providers and care managers in selecting the most relevant tests based on disease and/or ICD10 codes. The MBD Ranking System rates potential tests for any given disease and assigns an easily interpretable numerical and color-coded score based on clinical relevance, medical necessity, and testing indication (Figure 1). Tests with scores of 5 or above meet medical necessity, while those with scores of 4 or less do not. LDS ${ }^{\mathrm{TM}}$ follows Medicare's medical necessity guidelines by using testing indications such as "initial testing/ screening" to allow providers to better characterize the patient's disorder based on initial test results before ordering highly complex and/or expensive tests [2]. Within the system, each test is linked to its appropriate Current Procedural Terminology (CPT) code(s), and diseases, and each disease is linked to its applicable International Classification of Diseases (ICD)-10 and Systemized Nomenclature of Medicine (SNOMED) code. Accordingly, when LDS $^{\mathrm{TM}}$ solution is used to compliment an electronic medical record ordering system, the appropriate ICD10 and CPT codes can be sent directly with the order for use in claims submission (Figure 1A). In addition, LDS $^{\mathrm{TM}}$ has the capability to help select appropriate tests for more than one disease (co-morbidity) in a single order when clinical indications exist (Figure 1A). This platform offers a timely and relevant test utilization solution for physicians, hospitals and laboratory providers. It can also serve as an expert reference tool for payers in medical review, pre-approval and claims verification programs. Lastly, each test entry has content that describes test overview, test utility, interpretation, reference ranges, sample collection, handling and test methodology (Figure 1B). This information can be used as a supportive, educational and consultative tool for physician to better understand and interpret test results $[11,12]$. Since the platform can be interfaced with Electronic Medical Records (EMR), Electronic Hospital Records (EHR) and Laboratory Information Systems (LIS), the content can be access directly through these system and orders send directly to laboratories for testing. 


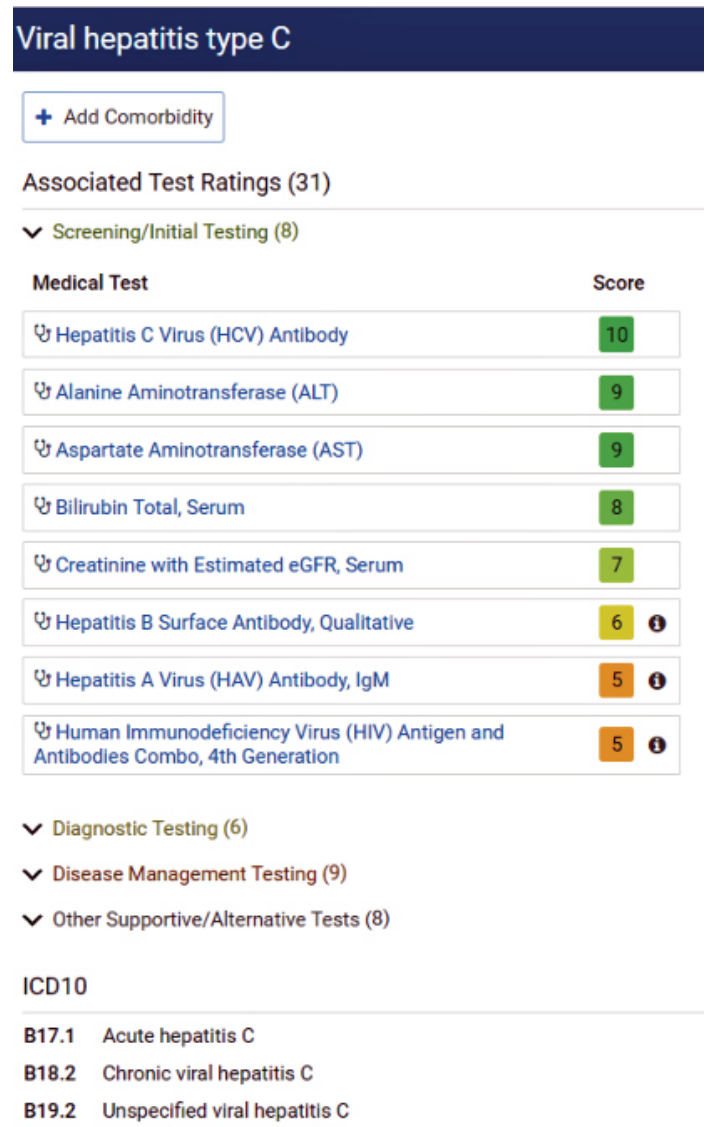

\section{Hepatitis C Virus (HCV) Antibody}

Associated Disease Ratings (58)
Screening/Initial Testing (5)
Disease
Miral hepatitis type C
Malignant neoplasm, liver
Adult preventative health screening
Prenatal care (Antenatal care)
Inclusion body myositis
Diagnostic Testing (47)
Disease Management Testing (6)
Overview
The hepatitis C virus or HCV is an infectious virus that affects the liver. HCV I
contact, blood to blood contact, sharing drug needles, and before 1990 throu
risk of liver cirrhosis and liver cancer. Symptoms of infection are fatigue, abd
majority of patients infected with the hepatitis C virus have little to no warnin
untreated will develop into acute phase hepatitis, which is typically within the
during this time frame.

B

Figure 1: Ordering test using LDS ${ }^{\mathrm{TM}} ; \mathbf{A}$ ) ordering by disease or ICD10. In this example "Viral hepatitis type $\mathrm{C}$ was used. As shown, most specific tests associated with the disease are listed and scored based on clinical relevance and medical necessity; B) ordering by test or procedure. In this case Hepatitis C Virus Antibody test was used. As shown, most common associated diseases with the test are listed and scored based on clinical relevance and medical necessity.

In order to qualify for reimbursement, every ordered test must be submitted with the correct ICD10 and CPT codes to indicate and support clinical relevance and medical necessity. The LDS ${ }^{\mathrm{TM}}$ platform can provide a comprehensive tool to assist billing staff and healthcare insurance payers with this process. The platform allows evidence and knowledge-based content to be queried by SNOMED, ICD10, CPT or disease/test description. These capabilities afford providers, payers and clinical lab service provider's further insight into the appropriateness of lab claim submission and reimbursement.

\section{Study supporting laboratory decisions system as a test utilization management}

To verify performance of this system, we analyzed claims or orders from a reference laboratory and a small insurance provider managing self-pay organizations. Claims or orders were reviewed using the MBD LDS $^{\mathrm{TM}}$ system for medical necessity based upon medical evidence score (based on clinical relevance and published guidelines) and based on Medicare Local and National Coverage Determination policies (LCDs and NCDs). The system has the capability to review every CPT and
ICD10 and compare to $\operatorname{LDS}^{\mathrm{TM}}$ medical evidence score and to CMS, LCD and NCD policies and make a recommendation.

\section{Reference Laboratory Study}

A total of 96,170 laboratory orders with almost 374,423 test claims were analyzed from a reference laboratory. The average number of HCPCS (CPT) codes per order was 3.8. Of these, 814 tests were accompanied by an invalid ICD10 (i.e., an ICD10 code that is not part of the most current ICD10 data set from CMS). There is a possibility that some of these "invalid" ICD10 codes actually were valid at the time of the order. Furthermore, 44,671 tests or $11.93 \%$ were accompanied by ICD10 codes that are described by Medicare as "never covered", often because they indicate that the test is performed for screening purposes and did not meet medical necessity. (https://www.cms.gov/Medicare/Coverage/ CoverageGenInfo/LabNCDsICD10.html). A total of 160,449 tests (i.e., $42.9 \%$ of total tests) were subject to an associated Medicare policy according to our findings. Based on these policies, 112,400 tests met coverage criteria and 48,049 tests did not. 
Of the original test claims sample, 342,699 tests (91.5\%) had an associated LDS ${ }^{\mathrm{TM}}$ score. Of these scored tests, 178,962 (47.80\%) met coverage and 163,737 (43.73\%) failed to meet coverage, according to the MBD LDS ${ }^{\mathrm{TM}}$ Ranking System. As stated above, the $\mathrm{LDS}^{\mathrm{TM}}$ can provide recommendations for alternative diagnostic ICD10 codes or tests which may aid physicians in choosing a more appropriate ICD10 diagnostic code or test. These recommended alternative ICD10 codes or tests are ones that would be covered according to the MBD LDS ${ }^{\mathrm{TM}}$ Ranking System and CMS LCDs and NCDs. Based on a subset composed of the first 10,000 claims reviewed, the LDS ${ }^{\mathrm{TM}}$ recommended 9,637 tests (96.4\%) with an alternative ICD10 code or test with a score above 5 , meeting medical necessity. Of these, 7755 tests (i.e., $80.5 \%$ ) were recommended by the $\mathrm{LDS}^{\mathrm{TM}}$ system which would meet Medicare policies, demonstrating that $\mathrm{LDS}^{\mathrm{TM}}$ system would correct inappropriate orders if employed as a testing utilization management system (Table 1).

\section{PPO Insurance Provider Managing Self-Pay Organizations Study}

In a second study, we analyzed 294,870 laboratory test claims from a PPO provider managing self-pay insurers seeking improvement in claim verification and cost reduction. Of these, 8 claims were missing ICD10 codes, 503 claims had invalid ICD10 codes, 30,017 (10.18\%) had ICD10 codes that were always denied, and 31,521 (10.69\%) were claims that Medicare would not support $(8.9 \%$ that did not meet NCDs and $1.79 \%$ that did not meet LCD policies). Of all submitted claims, 259,840 tests $(88.18 \%)$ were covered by the LDS ${ }^{\mathrm{TM}}$ system. Of those covered, $51.97 \%$ had LDS $^{\mathrm{TM}}$ scores $>5$ and the remaining $48.03 \%$ had scores $<5$. Interestingly, there were 4783 claims, or $1.62 \%$, for a 'General Health Panel' that Medicare and most of payers would not cover. If these had been eliminated, it would have alone represented a $\$ 228,388$ cost savings. Finally, $26.7 \%$ of claims were submitted with

Table 1: Independent Reference Laboratory claims analyzed for medical necessity by the LDS ${ }^{\mathrm{TM}}$.

\begin{tabular}{|c|c|c|}
\hline Claim Analytical Description & Number of Claims & Percentage \\
\hline Total orders & 96170 & \\
\hline Average \# HCPC per order & 3.833982 & \\
\hline Total tests & 374423 & \\
\hline \# of claims with SOME invalid ICD10 & 814 & $0.22 \%$ \\
\hline \# of claims with ALL NCD non-covered ICD10 & 44671 & $11.93 \%$ \\
\hline \# of claims with Medicare policy & 160449 & $42.85 \%$ \\
\hline \# of claims that Medicare supports & 112400 & $30.02 \%$ \\
\hline \# of claims that Medicare does not support & 48049 & $12.83 \%$ \\
\hline \# of claims that NCD does not support & 37505 & $10.02 \%$ \\
\hline \# of claims that LCD does not support & 10544 & $2.82 \%$ \\
\hline \# of claims with MDB score & 342699 & $91.53 \%$ \\
\hline \# of claims that MDB supports (score $>=5$ ) & 178962 & $47.80 \%$ \\
\hline \# of claims that MDB does not support $($ score $<5$ ) & 163737 & $43.73 \%$ \\
\hline \multicolumn{3}{|l|}{ First 10,000 claims subset } \\
\hline \# of claims with MDB test alternatives (score $>=5$ ) & 9637 & $96.37 \%$ \\
\hline \# of claims with MDB test alternatives that Medicare supports & 7755 & $80.47 \%$ \\
\hline
\end{tabular}

Table 2: Payer claim analyzed for medical necessity by the LDS ${ }^{\mathrm{TM}}$.

\begin{tabular}{|c|c|c|c|}
\hline Claim Analytical Description & Number of Claims & Percentage & Cost based on CMS \\
\hline Total claims & 294870 & & \\
\hline Total claims missing ICD10 & 8 & & \\
\hline Total claims with always-denied ICD10 & 30007 & $10.18 \%$ & \\
\hline Total claims with invalid ICD10 & 503 & $0.17 \%$ & \\
\hline \# of claims that Medicare does not support & 31528 & $10.69 \%$ & \\
\hline \# of claims that fail NCD & 26243 & $8.90 \%$ & \\
\hline \# of claims that fail LCD & 5285 & $1.79 \%$ & \\
\hline \# of claims with mdb determination/advice & 260020 & $88.18 \%$ & \\
\hline \# of claims with mdb support (score $\geq 5$ ) & 135141 & $51.97 \%$ & \\
\hline \# of claims with mdb does not support (score $<5$ ) & 124879 & $48.03 \%$ & \\
\hline \# of claims with mdb suggestions & 260020 & $100.00 \%$ & \\
\hline \# of claims with mdb suggestions $\geq 5$ & 259776 & $99.91 \%$ & \\
\hline General Health Panel & 4783 & $1.60 \%$ & $\$ 228,388.00$ \\
\hline All ICD10 Z codes & 77204 & $26.18 \%$ & $\$ 2,950,000.00$ \\
\hline ICD10 Z00.00 & 26052 & $8.84 \%$ & \\
\hline
\end{tabular}

Citation: Leblow L, Hamill T, Beqaj SH (2019) Use of Laboratory Decision System as a Test Utilization Management Tool in Clinical Settings, Current and Future Perspectives. J Clin Lab Med 4(1): dx.doi.org/10.16966/2572-9578.128 
a "Z" ICD10 code that would have required additional supportive ICD10 code(s) to establish medical necessity. If these claims were denied as not meeting medical necessity, the PPO would lose $\$ 2.95 \mathrm{M}$ in claims that could have been corrected had the LDS ${ }^{\mathrm{TM}}$ been used as an ordering system (Table 2).

Overall, in this study more than $50 \%$ of submitted orders did not meet medical necessity when compared to the MBD LDS ${ }^{\mathrm{TM}}$ Ranking System and more than 20\% did not meet Medicare LCD and NCD policies. Significant improvement would have resulted had the LDS ${ }^{\mathrm{TM}}$ been employed as a testing selection and ordering system because each order automatically would have been assigned the correct ICD10 and/ or diagnostic code. In addition, when appropriate, the $\mathrm{LDS}^{\mathrm{TM}}$ would make suggestions for more accurate ICD10 codes for every order, whether submitted by test, disease or ICD10 code. It is posited that the LDS $^{\mathrm{TM}}$ would improve orders more than 99\%; however, more analytical study is needed to support and verify this new MBD LDS ${ }^{\mathrm{TM}}$ system.

\section{Conclusion}

There is a clear and immediate need for a Laboratory Decision Support System similar to that which is used in Radiology which can aid providers in selecting the right test for each disease or condition, while assigning the correct ICD10 code to meet the medical necessity. Such an LDS ${ }^{\mathrm{TM}}$ system can assist providers in making appropriate utilization decisions while also supporting laboratories in reimbursement and streamline claim verification for payers, all of which combined will serve to make the laboratory industry and overall healthcare more efficient and cost-effective.

\section{References}

1. Jackson BR (2007) Managing laboratory test use: principles and tools. Clin Lab Med 27: 733-748.

2. Yeh DD (2014) A clinician's perspective on laboratory utilization management. Clin Chim Acta 427: 145-150.

3. Hickner J, Thompson PJ, Wilkinson T, Epner P, Sheehan M, et al. (2014) Primary care physicians' challenges in ordering clinical laboratory tests and interpreting results. J Am Board Fam Med 27: 268-274.

4. Debra Sherman (2012) Stemming the tide of overtreatment in U.S. healthcare. Health News. The American College of Physicians.

5. Huck A, Lewandrowski $K$ (2014) Utilization management in the clinical laboratory: an introduction and overview of the literature. Clin Chim Acta 427: 111-117.
6. Zhi M, Ding EL, Theisen-Toupal J, Whelan J, Arnaout R (2013) The landscape of inappropriate laboratory testing: a 15-year metaanalysis. PLoS One 8: e78962.

7. Gandhi TK, Kachalia A, Thomas EJ, Puopolo AL, Yoon C (2006) Missed and delayed diagnoses in the ambulatory setting: a study of closed malpractice claims. Ann Intern Med 145: 488-496.

8. Bindraban RS, Ten Berg MJ, Naaktgeboren CA, Kramer MHH, Van Solinge WW, et al. (2018) Reducing Test Utilization in Hospital Settings: A Narrative Review. Ann Lab Med 38: 402-412.

9. Bellodi E, Vagnoni E, Bonvento B, Lamma E (2017) Economic and organizational impact of a clinical decision support system on laboratory test ordering. BMC Med Inform Decis Mak 17: 179.

10. Faisal A, Andres K, Rind JAK, Das A, Alter D, et al. (2018) Reducing the number of unnecessary routine laboratory tests through education of internal medicine residents. Postgrad Med J 94: 716-719.

11. Plebani M, Aita A, Padoan A, Sciacovelli L (2019) Decision Support and Patient Safety. Clin Lab Med 39: 231-244.

12. Plebani M, Panteghini M (2014) Promoting clinical and laboratory interaction by harmonization. Clin Chim Acta 432: 15-21.

13. Konger RL, Ndekwe P, Jones G, Schmidt RP, Trey M, et al. (2016) Reduction in Unnecessary Clinical Laboratory Testing through Utilization Management at a US Government Veterans Affairs Hospital. Am J Clin Pathol 145: 355-364.

14. Kim JY, Dzik WH, Dighe AS, Lewandrowski KB (2011) Utilization Management in a Large Urban Academic Medical Center: a 10-year experience. Am J Clin Pathol 135: 108-118.

15. Burns J (2014) Health plans deploy new systems to control use of lab tests. Manag Care 23: 14,16-17,19-20.

16. Greenblatt MB, Nowak JA, Quade CC, Tanasijevic M, Lindeman N, et al. (2015) Impact of a Prospective Review Program for Reference Laboratory Testing Requests. Am J Clin Pathol 143: 627-634.

17. Dave Fornell (2017) CMS to Require Appropriate Use Criteria Documentation for Medical Imaging Orders. Diagnostic and Interventional Cardiology.

18. McMorris Rodgers, Cathy (2015-2016) H.R.2550 Medical Imaging Modernization Act of 2015. $114^{\text {th }}$ Congress.

19. Baron JM, Dighe AS (2014) The role of informatics and decision support in utilization management. Clin Chim Acta 427: 196-201.

20. Delvaux N, Van Thienen K, Heselmans A, de Velde SV, Ramaekers D, et al. (2017) The Effects of Computerized Clinical Decision Support Systems on Laboratory Test Ordering: A Systematic Review. Arch Pathol Lab Med 141: 585-595. 